\title{
Risk Factors for Anastomotic Leakage in Patients with Rectal Tumors Undergoing Anterior Resection within an ERAS Protocol: Results from the Swedish ERAS Database
}

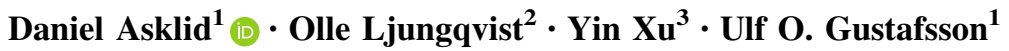

Accepted: 26 February 2021 / Published online: 17 March 2021

(C) The Author(s) 2021

\begin{abstract}
Background Research on risk factors for anastomotic leakage (AL) alone within an Enhanced Recovery After Surgery (ERAS) protocol has not yet been conducted. The aim of this study was to identify risk factors for AL and study short-term outcome after AL in patients operated with anterior resection (AR).

Methods All prospectively and consecutively recorded patients operated with AR in the Swedish part of the international ERAS ${ }^{\circledR}$ Interactive Audit System (EIAS) between January 2010 and February 2020 were included. The cohort was evaluated regarding risk factors for AL and short-term outcomes, including uni- and multivariate analysis. Pre-, intra- and postoperative compliance to ERAS ${ }^{\circledR}$ Society guidelines was calculated and evaluated.

Results Altogether 1900 patients were included, 155 (8.2\%) with AL and 1745 without AL. Male gender, obesity, peritoneal contamination, year of surgery 2016-2020, duration of primary surgery and age remained significant predictors for AL in multivariate analysis. There was no significant difference in overall pre- and intraoperative compliance to ERAS $®$ Society guidelines between groups. Only preadmission patient education remained as a significant ERAS variable associated with less AL. AL was associated with longer length of stay (LOS), higher morbidity rate and higher rate of reoperations.

Conclusion Male gender, obesity, peritoneal contamination, duration of surgery, surgery later in study period, age and preadmission patient education were associated with $\mathrm{AL}$ in patients operated on with AR. Overall pre- and intraoperative compliance to the ERAS protocol was high in both groups and not associated with AL.
\end{abstract}

Daniel Asklid

danielasklid@gmail.com

1 Department of Clinical Sciences, Division of Surgery, Danderyd Hospital, Karolinska Institute, Stockholm, Sweden

2 Department of Surgery, Örebro \& Institute of Molecular Medicine and Surgery, Örebro University and University Hospital, Karolinska Institute, Stockholm, Sweden

3 Clinical Epidemiology and Biostatistics, School of Medical Sciences, Örebro University, Örebro, Sweden

\section{Introduction}

Anastomotic leaks (AL) are detrimental to patients operated with rectal resection, conferring a large burden of morbidity, mortality and worse long-term oncological outcome [1-7]. Leak rates vary from 5 to $20 \%$, the highest rates reported in procedures involving total mesorectal excision (TME) [2,3]. The list of risk factors is wide and sometimes contradictory [2, 3, 8-11].

ERAS programs reduce surgical stress resulting in faster recovery, reduced postoperative morbidity/complications and shorter LOS and are gaining popularity worldwide [12-14]. Reports on potential associations between specific perioperative care items or compliance to the 
ERAS $®$ Society guideline protocol and AL specifically within an ERAS protocol have not yet been published.

Our main hypothesis was that compliance to the ERAS protocol, overall or in single intervention variables, has an effect on AL in patients operated with AR. The aim of the current study was to identify risk factors for AL, investigate the significance of compliance to the ERAS protocol in relation to AL, and to study outcome from surgery in AL and non-AL patients operated with AR due to rectal tumor within the Swedish part of the international ERAS database.

\section{Methods}

\section{Study design and setting}

This retrospective multicenter cohort study aimed to investigate potential predictors for AL in patients with rectal tumor (benign or malignant) operated with anterior resection. Compliance to the ERAS protocol and shortterm outcome for patients with AL were also investigated. The ERAS $®$ Society guideline for colorectal surgery consists of 25 evidence-based interventions [13] and all centers aim to treat their patients in agreement with this protocol. The international ERAS®Society Interactive Audit System (EIAS) database (EIAS) [15] was used as the source of data. EIAS assembles more than 90000 consecutive patients records, each with more than 300 variables and includes information on compliance to ERAS guidelines [13]. The Swedish part of the international ERAS database was validated in 2020 with excellent results regarding coverage, missing values and accuracy of data and used for the current study (submitted for publication).

Data on AL, perioperative variables, compliance to the ERAS protocol and outcome from surgery were collected from the EIAS database between January 1, 2010, and February 27, 2020. The definition of AL in EIAS is based on radiological diagnosis or intervention and/or reoperation.

The criteria set out in the Strengthening the Reporting of Observational studies in Epidemiology (STROBE) checklist was met conducting this publication. The study was approved by the Regional Ethical Review Board in Stockholm (2020-00,435).

\section{Participants}

All consecutive patients with benign or malignant rectal tumor $(N=1900)$ operated with open, laparoscopic or robotic $\mathrm{AR}$ and prospectively registered in the database were included in the study. Emergency surgery was not included. Rectal tumor was defined as a lesion within
$15 \mathrm{~cm}$ from anal verge. All tumor stages were included, and patients were analyzed according to intention-to-treat.

\section{Outcome variables}

Primary outcome was AL within 30 days from primary surgery. Secondary outcomes were possible consequences of AL: 30-day complications (Clavien I-II, $\geq$ III)[16], reoperation, LOS and death (30-day).

\section{Exposure variables}

Depending on availability of data in EIAS and results from prior research, the following variables were retained as potential risk factors in multivariate analysis based on purposeful selection modeling approach [17]: gender, age, body mass index, peritoneal soiling, preoperative radiotherapy, year of surgery, duration of primary surgery and preadmission patient education.

\section{Data analysis}

No formal sample size calculation was conducted since secondary data were used, but it is feasible to consider power to detect a meaningful effect. At the 5\% significance level, the sample size allows for the detection of an odds ratio $>1.7$ for a binary predictor variable with $80 \%$ power [18].

Both unadjusted and adjusted models were performed. Chi-squared test or Fisher exact test (for categorical variables with cell size less than 5) was performed to test unadjusted associations between categorical variables in basic characteristics (Table 1), intraoperative variables (Table 2), pre- and intraoperative interventions (Table 3), postoperative compliance variables (Table 4), secondary outcomes and AL. Wilcoxon's rank sum test was performed to test the unadjusted association between continuous variables (Table 1, 2, 3, 4 and LOS) and AL.

Multivariate logistic regression was estimated based on purposeful selection modeling approach [17] from basic characteristics, intraoperative variables, and pre- and intraoperative compliance rate. Potential risk factors with $p<0.25$ in the unadjusted models were included in the initial step of multivariate logistic regression; then risk factors with $p>0.1$ and a change in any remaining parameter estimates $<15 \%$ compared to the full model (the initial step) were successively removed. Variables included in multivariable analyses had $0.1 \%-37.9 \%$ missing information. This was handled via multiple imputation using iterative chained equations [19].

Normal distribution was tested using Shapiro-Francia test. Compliance data were calculated as the numbers of achieved interventions divided with the total number of 
Table 1 Basic characteristics stratified by anastomotic leakage

\begin{tabular}{|c|c|c|c|c|}
\hline & \multicolumn{2}{|c|}{ Anastomotic leakage } & \multirow[b]{2}{*}{ Unadjusted analysis } & \multirow[b]{2}{*}{ Adjusted analysis } \\
\hline & No $(N=1745)$ & Yes $(N=155)$ & & \\
\hline Sex & & & $\chi^{2}(1)=15.08, p<0.001$ & $1.91(1.30,2.79)$ \\
\hline Male & $992(56.9)$ & $113(72.9)$ & & \\
\hline Female (reference group) & $753(43.1)$ & $42(27.1)$ & & \\
\hline Missing & $0(0.0)$ & $0(0.0)$ & & \\
\hline Age (years) & & & $Z=3.64, p<0.001$ & $0.98(0.96,0.99)$ \\
\hline$N$ & 1743 & 155 & & \\
\hline Missing $N$ & $2(0.1)$ & $0(0.0)$ & & \\
\hline Mean (SD) & $68.09(11.0)$ & $64.99(10.6)$ & & \\
\hline Year of operation & & & $\chi^{2}(1)=12.81, p<0.001$ & $1.84(1.22,2.79)$ \\
\hline 2010-2015 (reference group) & $645(37.0)$ & $35(22.6)$ & & \\
\hline $2016-2020$ & $1100(63.0)$ & $120(77.4)$ & & \\
\hline Missing & $0(0.0)$ & $0(0.0)$ & & \\
\hline Preoperative WHO score & & & $p=0.060$ & \\
\hline Asymptomatic & $1267(72.6)$ & $121(78.1)$ & & \\
\hline Symptomatic but completely ambulatory & $233(13.4)$ & $14(9.0)$ & & \\
\hline Symptomatic, $<50 \%$ in bed during the day & $25(1.4)$ & $0(0.0)$ & & \\
\hline Symptomatic, $>50 \%$ in bed & $2(0.1)$ & $1(0.7)$ & & \\
\hline Missing & $218(12.5)$ & $19(12.2)$ & & \\
\hline Cancer & & & $p=0.237$ & \\
\hline No & $87(5.0)$ & $4(2.6)$ & & \\
\hline Yes & $1658(95.0)$ & $151(97.4)$ & & \\
\hline Missing & $0(0.0)$ & $0(0.0)$ & & \\
\hline Preoperative nutritional treatment & & & $\chi^{2}(1)=0.58, p=0.445$ & \\
\hline No & $1244(71.3)$ & $122(78.7)$ & & \\
\hline Yes & $190(10.9)$ & $15(9.7)$ & & \\
\hline Missing & $311(17.8)$ & $18(11.6)$ & & \\
\hline Preoperative nutritional status & & & $p=0.464$ & \\
\hline Normal status & $1094(62.7)$ & $107(69.0)$ & & \\
\hline Risk of malnutrition & $205(11.8)$ & $15(9.7)$ & & \\
\hline Malnourished & $15(0.8)$ & $2(1.3)$ & & \\
\hline Missing & $431(24.7)$ & $31(20.0)$ & & \\
\hline Smoking & & & $\chi^{2}(2)=3.71, p=0.157$ & \\
\hline No & $1540(88.2)$ & $123(79.3)$ & & \\
\hline Stopped due to surgery & $53(3.0)$ & $8(5.2)$ & & \\
\hline Yes & $76(4.4)$ & $9(5.8)$ & & \\
\hline Missing & $76(4.4)$ & $15(9.7)$ & & \\
\hline Alcohol & & & $\chi^{2}(2)=2.97, p=0.226$ & \\
\hline No & 1027 (58.9) & $90(58.1)$ & & \\
\hline Stopped due to surgery & $64(3.7)$ & $5(3.2)$ & & \\
\hline Yes & $55(3.2)$ & $9(5.8)$ & & \\
\hline Missing & $599(34.2)$ & $51(32.9)$ & & \\
\hline Recreational drug use & & & $\chi^{2}(1)=2.87, p=0.090$ & \\
\hline No & 1042 (59.7) & $104(67.1)$ & & \\
\hline Yes & $28(1.6)$ & $6(3.9)$ & & \\
\hline Missing & $675(38.7)$ & $45(29.0)$ & & \\
\hline Diabetes & & & $\chi^{2}(1)=0.23, p=0.633$ & \\
\hline No & $1505(86.3)$ & $131(84.5)$ & & \\
\hline
\end{tabular}


Table 1 continued

\begin{tabular}{|c|c|c|c|c|}
\hline & \multicolumn{2}{|c|}{ Anastomotic leakage } & \multirow[b]{2}{*}{ Unadjusted analysis } & \multirow[b]{2}{*}{ Adjusted analysi } \\
\hline & No $(N=1745)$ & Yes $(N=155)$ & & \\
\hline Yes & $236(13.5)$ & $23(14.8)$ & & \\
\hline Missing & $4(0.2)$ & $1(0.7)$ & & \\
\hline BMI $\left(\mathrm{kg} / \mathrm{m}^{2}\right)$ & & & $p=0.044$ & $1.37(0.30,6.17)$ \\
\hline Under weight, 15 to $<18.5$ & $28(1.6)$ & $2(1.3)$ & & $1.16(0.78,1.72)$ \\
\hline Normal weight (reference group), 18.5 to $<25$ & $714(40.9)$ & $48(31.0)$ & & $1.62(1.01,2.62)$ \\
\hline Over weight, 25 to $<30$ & $714(40.9)$ & $66(42.6)$ & & \\
\hline Obese, $\geq 30$ & $263(15.1)$ & $34(21.9)$ & & \\
\hline Missing & $26(1.5)$ & $5(3.2)$ & & \\
\hline ASA physical status & & & $p=0.533$ & \\
\hline 1 (reference group) & $331(19.0)$ & $36(23.2)$ & & \\
\hline 2 & $992(56.9)$ & $85(54.8)$ & & \\
\hline 3 & $383(22.0)$ & $31(20.1)$ & & \\
\hline 4 & $14(0.8)$ & $0(0.0)$ & & \\
\hline 5 & $1(0.1)$ & $0(0.0)$ & & \\
\hline Missing & $24(1.4)$ & $3(1.9)$ & & \\
\hline Severe heart disease & & & $\chi^{2}(1)=1.15, p=0.284$ & \\
\hline No & $1128(64.6)$ & $114(73.6)$ & & \\
\hline Yes & $76(4.4)$ & $11(7.1)$ & & \\
\hline Missing & $541(31.0)$ & $30(19.3)$ & & \\
\hline Severe pulmonary disease & & & $\chi^{2}(1)=1.01, p=0.314$ & \\
\hline No & $1176(67.4)$ & $120(77.4)$ & & \\
\hline Yes & $30(1.7)$ & $5(3.2)$ & & \\
\hline Missing & $539(30.9)$ & $30(19.4)$ & & \\
\hline Preoperative chemotherapy & & & $\chi^{2}(1)=0.004, p=0.950$ & \\
\hline No & $1535(88.0)$ & $137(88.4)$ & & \\
\hline Yes & $205(11.8)$ & $18(11.6)$ & & \\
\hline Missing & $5(0.2)$ & $0(0.0)$ & & \\
\hline Preoperative radiotherapy & & & $\chi^{2}(1)=5.64, p=0.018$ & $1.35(0.96,1.91)$ \\
\hline No (reference group) & $1012(58.0)$ & $75(48.4)$ & & \\
\hline Yes & $726(41.6)$ & $80(51.6)$ & & \\
\hline Missing & $7(0.4)$ & $0(0.0)$ & & \\
\hline Previous surgery to the abdominal region & & & $\chi^{2}(1)=1.31, p=0.252$ & \\
\hline No & $1297(74.3)$ & $123(79.4)$ & & \\
\hline Yes & $427(24.5)$ & $32(20.6)$ & & \\
\hline Missing & $21(1.2)$ & $0(0.0)$ & & \\
\hline Preadmission stoma counselling & & & $\chi^{2}(1)=0.03, p=0.861$ & \\
\hline No & $128(7.3)$ & $14(9.0)$ & & \\
\hline Yes & $1050(60.2)$ & $109(70.3)$ & & \\
\hline Missing & $567(32.5)$ & $32(20.7)$ & & \\
\hline
\end{tabular}

Uni- and multivariate analysis

$\chi^{2}$ test or Fisher exact test (for categorical variables with cell size less than 5) was performed to test the unadjusted association between each categorical basic characteristic listed and anastomotic leakage. For difference in age, Wilcoxon's rank sum test was performed

Values in parenthesis are percentages, except in the column for unadjusted associations, here values in parenthesis = degrees of freedom. ASA (American Society of Anesthesiologists physical status), BMI (body mass index)

Odds ratios and $95 \%$ confidence interval were reported for adjusted model which includes measures of, gender, age, body mass index, peritoneal soiling, preoperative radiotherapy, year of surgery, duration of primary surgery (hours) and preadmission patient education given (Table 3) 
Table 2 Intraoperative variables stratified by anastomotic leakage

\begin{tabular}{|c|c|c|c|c|}
\hline & \multicolumn{2}{|c|}{ Anastomotic leakage } & \multirow[b]{2}{*}{ Unadjusted analysis } & \multirow[b]{2}{*}{ Adjusted analysis } \\
\hline & No $(N=1745)$ & Yes $(N=155)$ & & \\
\hline Surgical procedure & & & $\chi^{2}(1)=7.61, p=0.022$ & \\
\hline Robotic (reference group) & $460(26.3)$ & $56(36.1)$ & & \\
\hline Open & $774(44.4)$ & $55(35.5)$ & & \\
\hline Laparoscopic & $511(29.3)$ & $44(28.4)$ & & \\
\hline Missing & $0(0.0)$ & $0(0.0)$ & & \\
\hline Additional procedures & & & $\chi^{2}(1)=0.46, p=0.497$ & \\
\hline No (reference group) & $1141(65.4)$ & $117(75.5)$ & & \\
\hline Yes & $134(7.7)$ & $11(7.1)$ & & \\
\hline Missing & $470(26.9)$ & $27(17.4)$ & & \\
\hline Peritoneal soiling/contamination & & & $\chi^{2}(1)=9.22, p=0.002$ & \\
\hline No (reference group) & $1566(89.7)$ & $121(78.1)$ & & \\
\hline Yes & $104(6.0)$ & 18 (11.6) & & $1.79(1.02,3.14)$ \\
\hline Missing & $75(4.3)$ & $16(10.3)$ & & \\
\hline Systemic opioids given during surgery & & & $\chi^{2}(1)=2.08, p=0.149$ & \\
\hline No & $231(13.2)$ & $17(11.0)$ & & \\
\hline Yes & $976(55.9)$ & $106(68.4)$ & & \\
\hline Missing & $538(30.9)$ & $32(20.6)$ & & \\
\hline Depth of anesthesia monitored & & & $\chi^{2}(1)=1.59, p=0.207$ & \\
\hline No & $343(19.7)$ & $28(18.1)$ & & \\
\hline Yes & $887(50.8)$ & $96(61.9)$ & & \\
\hline Missing & $515(29.5)$ & $31(20.0)$ & & \\
\hline Pre- and intraoperative ERAS compliance rate $(\%)$ & & & $Z=0.82, p=0.410$ & \\
\hline $\mathrm{N}$ & $1549(88.8)$ & $137(88.4)$ & & \\
\hline Missing N & $196(11.2)$ & 18 (11.6) & & \\
\hline Mean (SD) & $93.15(7.91)$ & $92.66(8.94)$ & & \\
\hline Duration of primary surgery (hours) & & & $Z=-5.21, p<0.001$ & $1.13(1.03,1.23)$ \\
\hline $\mathrm{N}$ & $1732(99.3)$ & $151(97.4)$ & & \\
\hline Missing $\mathrm{N}$ & $13(0.7)$ & $4(2.6)$ & & \\
\hline Mean (SD) & $4.64(1.69)$ & $5.48(2.22)$ & & \\
\hline New ileostomy & & & $\chi^{2}(1)=5.23, p=0.022$ & \\
\hline No & $739(42.3)$ & $51(32.9)$ & & \\
\hline Yes & $1006(57.7)$ & $104(67.1)$ & & \\
\hline Missing & $0(0.0)$ & $0(0.0)$ & & \\
\hline
\end{tabular}

Univariate and multivariate analysis

$\chi^{2}$ test or Fisher exact test (for categorical variables with cell size less than 5) was performed to test unadjusted association between each intraoperative variable and anastomotic leakage. For difference in ERAS compliance rate and duration of primary surgery, Wilcoxon's rank sum test was performed

Values in parenthesis are percentages if not stated otherwise, except in the column for unadjusted associations, here values in parenthesis $=$ degrees of freedom

Odds ratios and $95 \%$ confidence interval were reported for adjusted analysis which includes measures of, gender, age, body mass index, peritoneal soiling, preoperative radiotherapy, year of surgery, duration of surgery (hours) and preadmission patient education

pre- and intraoperative interventions. To avoid bias, only compliance to pre- and intraoperative interventions was analyzed, since postoperative interventions could be considered as variables related to the outcome of surgery [20].
Conversion to open surgery was analyzed on intentionto-treat basis.

Categorical variables were presented as frequencies and percentage and continuous variables as mean with standard deviation (SD) or median with interquartile range when 
Table 3 Preoperative and intraoperative compliance stratified by anastomotic leakage

\begin{tabular}{|c|c|c|c|}
\hline & \multicolumn{2}{|c|}{ Anastomotic leakage } & \multirow[b]{2}{*}{ Unadjusted association } \\
\hline & No $(N=1745)$ & Yes $(N=155)$ & \\
\hline \multicolumn{4}{|l|}{ Preoperative compliance } \\
\hline Preadmission patient education given & & & $\chi^{2}(1)=2.24, p=0.134$ \\
\hline Non-compliant & $52(3.0)$ & $8(5.2)$ & \\
\hline Compliant & $1687(96.7)$ & $146(94.2)$ & \\
\hline Missing & $6(0.3)$ & $1(0.6)$ & \\
\hline Preoperative oral carbohydrate treatment & & & $\chi^{2}(1)=2.03, p=0.155$ \\
\hline Non-compliant & $79(4.5)$ & $11(7.1)$ & \\
\hline Compliant & $1610(92.3)$ & $140(90.3)$ & \\
\hline Missing & $56(3.2)$ & $4(2.6)$ & \\
\hline Mechanical bowel preparation & & & - \\
\hline Non-compliant & $0(0.0)$ & $0(0.0)$ & \\
\hline Compliant & $387(22.2)$ & $21(13.6)$ & \\
\hline Missing & $12(0.7)$ & $1(0.6)$ & \\
\hline Not applicable & $1346(77.1)$ & $133(85.8)$ & \\
\hline Preoperative long-acting sedative medication & & & $\chi^{2}(1)=0.08, p=0.779$ \\
\hline Non-compliant & $240(13.8)$ & $22(14.2)$ & \\
\hline Compliant & $1459(83.6)$ & $125(80.6)$ & \\
\hline Missing & $46(2.6)$ & $8(5.2)$ & \\
\hline Antibiotic prophylaxis & & & 0.394 \\
\hline Non-compliant & $19(1.1)$ & $0(0.0)$ & \\
\hline Compliant & $1721(98.6)$ & $153(98.7)$ & \\
\hline Missing & $5(0.3)$ & $2(1.3)$ & \\
\hline Thrombosis prophylaxis & & & $\chi^{2}(1)=0.02, p=0.891$ \\
\hline Non-compliant & $64(3.7)$ & $6(3.9)$ & \\
\hline Compliant & $1676(96.0)$ & $148(95.5)$ & \\
\hline Missing & $5(0.3)$ & $1(0.6)$ & \\
\hline PONV prophylaxis administered & & & 0.725 \\
\hline Non-compliant & $43(2.5)$ & $1(0.7)$ & \\
\hline Compliant & $738(42.3)$ & $43(27.7)$ & \\
\hline Missing & $8(0.5)$ & $1(0.6)$ & \\
\hline Not applicable & $956(54.7)$ & $110(71.0)$ & \\
\hline \multicolumn{4}{|l|}{ Intraoperative compliance } \\
\hline Infusion of vasoactive drugs & & & $\chi^{2}(1)=1.46, p=0.227$ \\
\hline Non-compliant & $436(25.0)$ & $32(20.7)$ & \\
\hline Compliant & $1242(71.2)$ & $117(75.5)$ & \\
\hline Missing & $67(3.8)$ & $6(3.8)$ & \\
\hline Upper-body forced-air heating cover used & & & 1.000 \\
\hline Non-compliant & $46(2.6)$ & $4(2.6)$ & \\
\hline Compliant & $1667(95.5)$ & $149(96.1)$ & \\
\hline Missing & $32(1.9)$ & $2(1.3)$ & \\
\hline Total IV volume of fluids intraoperatively & & & $\chi^{2}(1)=4.62, p=0.032$ \\
\hline Non-compliant & $63(3.6)$ & $11(7.1)$ & \\
\hline Compliant & $1682(96.4)$ & $144(92.9)$ & \\
\hline Missing & $0(0.0)$ & $0(0.0)$ & \\
\hline Preoperative compliance rate $(\%)$ & & & $Z=0.97, p=0.332$ \\
\hline $\mathrm{N}$ & $1617(92.7)$ & $142(91.6)$ & \\
\hline Missing $N$ & $128(7.3)$ & $13(8.4)$ & \\
\hline
\end{tabular}


Table 3 continued

\begin{tabular}{|c|c|c|c|}
\hline & \multicolumn{2}{|c|}{ Anastomotic leakage } & \multirow[b]{2}{*}{ Unadjusted association } \\
\hline & No $(N=1745)$ & Yes $(N=155)$ & \\
\hline Mean (SD) & $95.14(9.04)$ & $94.34(10.18)$ & \\
\hline Intraoperative compliance rate $(\%)$ & & & $Z=-0.45, p=0.651$ \\
\hline$N$ & $1654(94.8)$ & $149(96.1)$ & \\
\hline Missing $N$ & $91(5.2)$ & $6(3.9)$ & \\
\hline Mean (SD) & $89.16(17.10)$ & $89.49(17.79)$ & \\
\hline
\end{tabular}

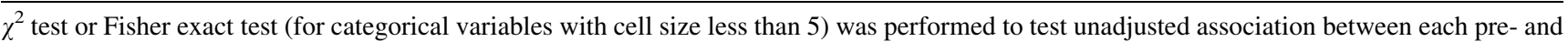
intra-operative compliance (compliant vs non-compliant) and anastomotic leakage. No Fisher exact test was calculated for oral bowel preparation since dichotomous responses were not present (the entire row for non-compliant was zero). For difference in compliance rate, Wilcoxon's rank sum test was performed. Values in parenthesis are percentages if not stated otherwise, except in the column for unadjusted associations, here values in parenthesis $=$ degrees of freedom

Table 4 Postoperative compliance stratified by anastomotic leakage

\begin{tabular}{|c|c|c|c|}
\hline & \multicolumn{2}{|l|}{ Anastomotic leakage } & \multirow[b]{2}{*}{ Unadjusted association } \\
\hline & No $(N=1745)$ & Yes $(N=155)$ & \\
\hline Opioid use-On day of surgery & & & $x^{2}(1)=4.98, p=0.026$ \\
\hline Non-compliant & $741(42.5)$ & $88(56.8)$ & \\
\hline Compliant & $479(27.4)$ & $36(23.2)$ & \\
\hline Missing & $525(30.1)$ & $31(20.0)$ & \\
\hline Opioid use-On POD 1 & & & $x^{2}(1)=15.84, p<0.001$ \\
\hline Non-compliant & $687(39.4)$ & $93(60.0)$ & \\
\hline Compliant & $529(30.3)$ & $31(20.0)$ & \\
\hline Missing & $529(30.3)$ & $31(20.0)$ & \\
\hline Opioid use-On POD 2 & & & $x^{2}(1)=13.05, p<0.001$ \\
\hline Non-compliant & $729(41.8)$ & $94(60.7)$ & \\
\hline Compliant & $466(26.7)$ & $27(17.4)$ & \\
\hline Missing & $550(31.5)$ & $34(21.9)$ & \\
\hline Opioid use-On POD 3 & & & $x^{2}(1)=14.87, p<0.001$ \\
\hline Non-compliant & $710(40.7)$ & $96(61.9)$ & \\
\hline Compliant & $431(24.7)$ & $24(15.5)$ & \\
\hline Missing & $604(34.6)$ & $35(22.6)$ & \\
\hline Total IV volume of fluids day $0(\mathrm{~mL})$ & & & $Z=-1.52, p=0.129$ \\
\hline$N$ & 1745 & 155 & \\
\hline Mean (SD) & $2869.79(1601.67)$ & $3124.64(1804.02)$ & \\
\hline Missing & $0(0.0)$ & $0(0.0)$ & \\
\hline Duration of IV fluid infusion (days) & & & $Z=-2.83, p=0.005$ \\
\hline$N$ & 1701 & 148 & \\
\hline Mean (SD) & $1.95(4.02)$ & $3.51(6.46)$ & \\
\hline Missing & $44(2.5)$ & $7(4.5)$ & \\
\hline Time to passage of flatus (days) & & & $Z=-1.47, p=0.143$ \\
\hline$N$ & 1453 & 117 & \\
\hline Mean (SD) & $1.63(1.85)$ & $1.97(2.18)$ & \\
\hline Missing & $292(16.7)$ & $38(24.5)$ & \\
\hline First passage of stool (days) & & & $Z=-0.72, p=0.473$ \\
\hline$N$ & 1652 & 142 & \\
\hline Mean (SD) & $2.42(3.58)$ & $3.09(4.53)$ & \\
\hline
\end{tabular}


Table 4 continued

\begin{tabular}{|c|c|c|c|}
\hline & \multicolumn{2}{|c|}{ Anastomotic leakage } & \multirow[b]{2}{*}{ Unadjusted associatior } \\
\hline & No $(N=1745)$ & Yes $(N=155)$ & \\
\hline Missing & $93(5.33)$ & $13(8.39)$ & \\
\hline Time to tolerating solid food (days) & & & $Z=-5.18, p<0.001$ \\
\hline$N$ & 1546 & 122 & \\
\hline Mean (SD) & $3.13(4.78)$ & $5.95(7.25)$ & \\
\hline Missing & $199(11.4)$ & $33(21.3)$ & \\
\hline Termination of urinary drainage (days) & & & $Z=-4.43, p<0.001$ \\
\hline$N$ & 1497 & 119 & \\
\hline Mean (SD) & $4.51(7.21)$ & $7.84(8.88)$ & \\
\hline Missing & $248(14.2)$ & $36(23.2)$ & \\
\hline Time to pain control with oral analgetics (days) & & & $Z=-4.28, p<0.001$ \\
\hline$N$ & 1660 & 133 & \\
\hline Mean (SD) & $3.63(4.28)$ & $5.44(4.85)$ & \\
\hline Missing & $85(4.87)$ & $22(14.19)$ & \\
\hline
\end{tabular}

$x^{2}$ test or Fisher exact test (for categorical variables with cell size less than 5) was performed to test the difference in post-operative compliance for categorical variables, whereas, Wilcoxon's rank sum test was performed for continuous variables

Values in parenthesis are percentages if not stated otherwise, except in the column for unadjusted associations, here values in parenthesis $=$ degrees of freedom

appropriate. Results from multivariate logistic analysis were reported as OR and $95 \% \mathrm{CI}$.

A $p$ value $<0.05$ or $95 \%$ CI not including 1 was considered statistically significant. Stata version 16.0 (StataCorp, College Station, Texas, United States of America) was used for statistical analysis.

\section{Results}

In all, 1900 patients were included in the study, 155 (8.2\%) with AL. Of all AL, 54 (34.8\%) had a radiological diagnosis only, without intervention, $13(8.4 \%)$ had a percutaneous drain only, and $88(56.8 \%)$ had a reoperation.

\section{Time, leak rate and surgical approach}

Figure 1 illustrates AL rate over time stratified on surgical approach. In 2013, only 4 robotic operations were performed, and one suffered a leak. The frequency of AL increased over time for all modalities and surgery late in the study period (2016-2020) was a risk factor for AL (9.8\% vs $5.1 \%$, OR $1.84,95 \%$ CI $(1.22,2.79))$ compared to surgery early in the study period (2010-2015), demonstrated in Table 1.

\section{Basic characteristics and intraoperative variables}

Basic characteristics stratified by AL are shown in Table 1. Males had a higher AL rate than females (10.2\% vs $5.3 \%$, OR $1.91,95 \%$ CI $(1.30,2.79))$. Obesity was a risk factor for $\mathrm{AL}(11.5 \%$ vs $6.3 \%$, OR 1.62, 95\% CI $(1.01,2.62))$ compared to normal weight. Although preoperative radiotherapy was a significant risk factor for $\mathrm{AL}$ in univariate analysis, this association did not remain in the multivariate model, OR 1.29 (0.91, 1.83).

Patients with peritoneal contamination had a higher AL rate compared to patients without contamination $(14.8 \%$ vs $7.2 \%$, OR 1.79, 95\% CI $(1.02,3.14))$, as shown in Table 2. Duration of primary surgery and age were both associated with AL OR 1.13, 95\% CI $(1.03,1.23)$ and OR 0.98, 95\% CI $(0.96,0.99)$ respectively.

\section{Compliance to the ERAS protocol}

Pre- and intraoperative compliance measures to the ERAS protocol are shown in Table 3. Comparing unadjusted AL rates in compliant vs non-compliant, one item-total iv volume of fluids intraoperatively, showed significant difference in favor of the compliant group $\left(7.9 \%\right.$ vs $14.9 \%, \chi^{2}$ (1) $=4.62, p=0.032$ ); however, fluid management failed to reach significance in the multivariate logistic regression. The reverse was shown for preadmission patient education, which was not significant in univariate analysis but showed to be an independent protective factor for $\mathrm{AL}$ after 


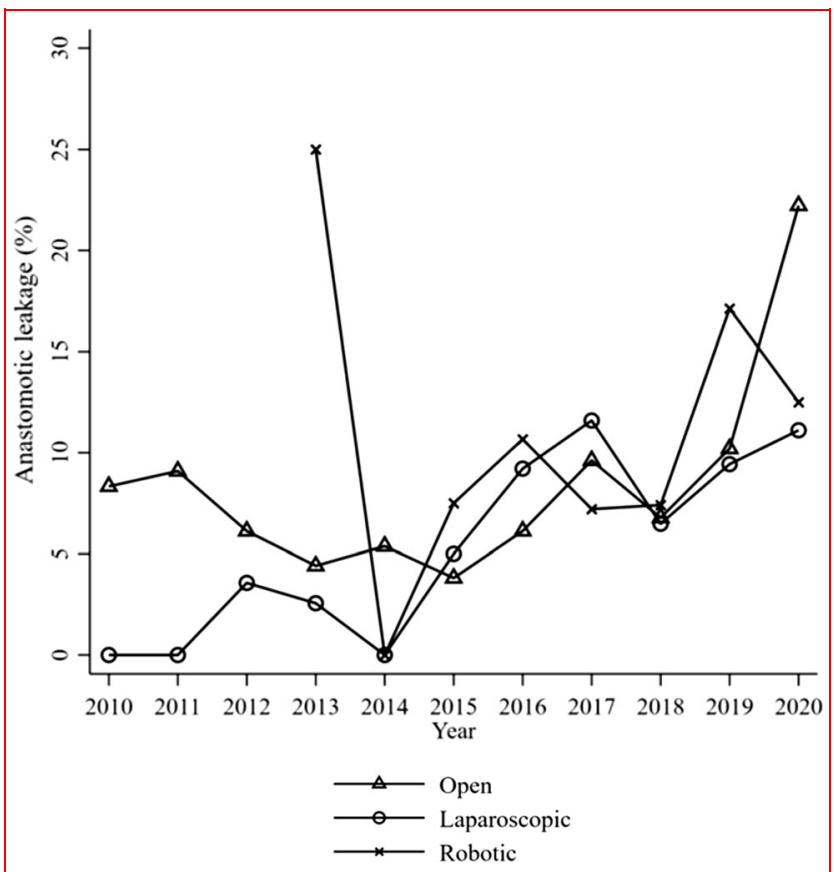

Fig. 1 Anastomotic leakage over time stratified by surgical approach

adjustment (OR 0.41, 95\% CI (0.19. 0.92). Overall, mean (SD) pre- and intraoperative compliance rates were high in both the AL group (92.66\% (8.94)) and the no AL group (93.15\% (7.91)) and showed no significant differences in unadjusted $(\mathrm{Z}=0.82, p=0.410)$ or adjusted (OR 0.99, $95 \%$ CI $(0.97,1.01))$ analysis. Patients suffering from AL demonstrated significantly worse postoperative compliance (Table 4).

\section{Short-term outcome}

Major complications (Clavien-Dindo $\geq 3$ ) were more common in patients with AL (63.9\% vs 5.9\%, $p<0.001)$. Results favored the non-AL group regarding reoperations $\left(6.6 \%\right.$ vs $\left.69.7 \%, \chi^{2}(1)=542.68, p<0.001\right)$ and LOS (median 7 vs 15 nights, $\mathrm{Z}=-11.72, p<0.001$ ). No difference was observed in postoperative mortality (30 days). Selected complications stratified by $\mathrm{AL}$ are shown in Fig. 2.

\section{Discussion}

In this large multicenter cohort study within an ERAS protocol, male gender, time of surgery, obesity, longer duration of surgery, peritoneal contamination, age and preadmission education were found to be independent predictors of anastomotic leakage in patients with rectal tumor operated with anterior resection. Overall compliance to pre- and intraoperative interventions in the ERAS protocol was high regardless of AL and did not affect the risk of AL. When evaluating single compliance items, total iv volume of fluids intraoperatively demonstrated significantly better compliance in patients without AL in univariate analysis. However, the variable did not qualify as an independent risk factor after adjusting for other covariates. After adjustment, only preadmission education remained as a protective ERAS compliance variable.

Results diverge in the literature on risk factors for $\mathrm{AL}$ $[2,3,8,21]$. This may partly be explained by factors such as heterogeneity in patient recruitment, lack of description regarding perioperative treatment protocols differences in follow-up and underpowered studies. Furthermore, a lack of international consensus on how to define $\mathrm{AL}$, with a vast variability in terminology and grading terms resulting in uncertainty on how to clinically diagnose AL (by symptoms, radiography or reoperation), makes a comparison between different studies difficult.

In 2010, guidelines defining and grading AL following rectal surgery was published [22] and later validated [23]. $\mathrm{AL}$ was in the guidelines defined as a "defect of the intestinal wall at the anastomotic site leading to a communication between the intra- and extraluminal compartments" and graded according to Grade A, Grade B and C depending on severity of AL.

Despite these efforts made, surgeons still have different opinions regarding definitions of $\mathrm{AL}$ and what actions are needed in order to treat AL.

The EIAS definition of AL is either radiological findings and/or a reoperation, to some extent contribute to the general variability between studies. However, the definition is identical for all centers recording in the database, and thus, all AL were recorded according to the same terms. AL rate in the current study was $8.2 \%$, in line with previous results from literature where compliance to ERAS elements is unknown $[2,3]$. The majority were leaks which required reoperation. However, since about $30 \%$ of $\mathrm{AL}$ are diagnosed beyond 30 days after surgery, the true leakage rate in the present study is likely to be underestimated [24].

The multivariate analysis identified male gender, obesity, peritoneal contamination, time of surgery, duration of surgery, age and preadmission education as independent predictors for AL. The majority of these predictors are previously reported, for example male gender due to a narrow pelvis, more difficult surgery $[25,26]$ or hormonerelated differences compared to females impeding microcirculation [27]. The literature also supports the relationship between high BMI and AL [2, 3, 8], explained by more difficult surgery and a larger burden of comorbidities in obese patients. Intraoperative contamination is also a recognized risk factor for AL [28]. Operation time is 
Fig. 2 Selected complications (\%) stratified by AL. *P value less than 0.05 was considered significant. Further 12

complications were compared (not shown) without significant difference between groups

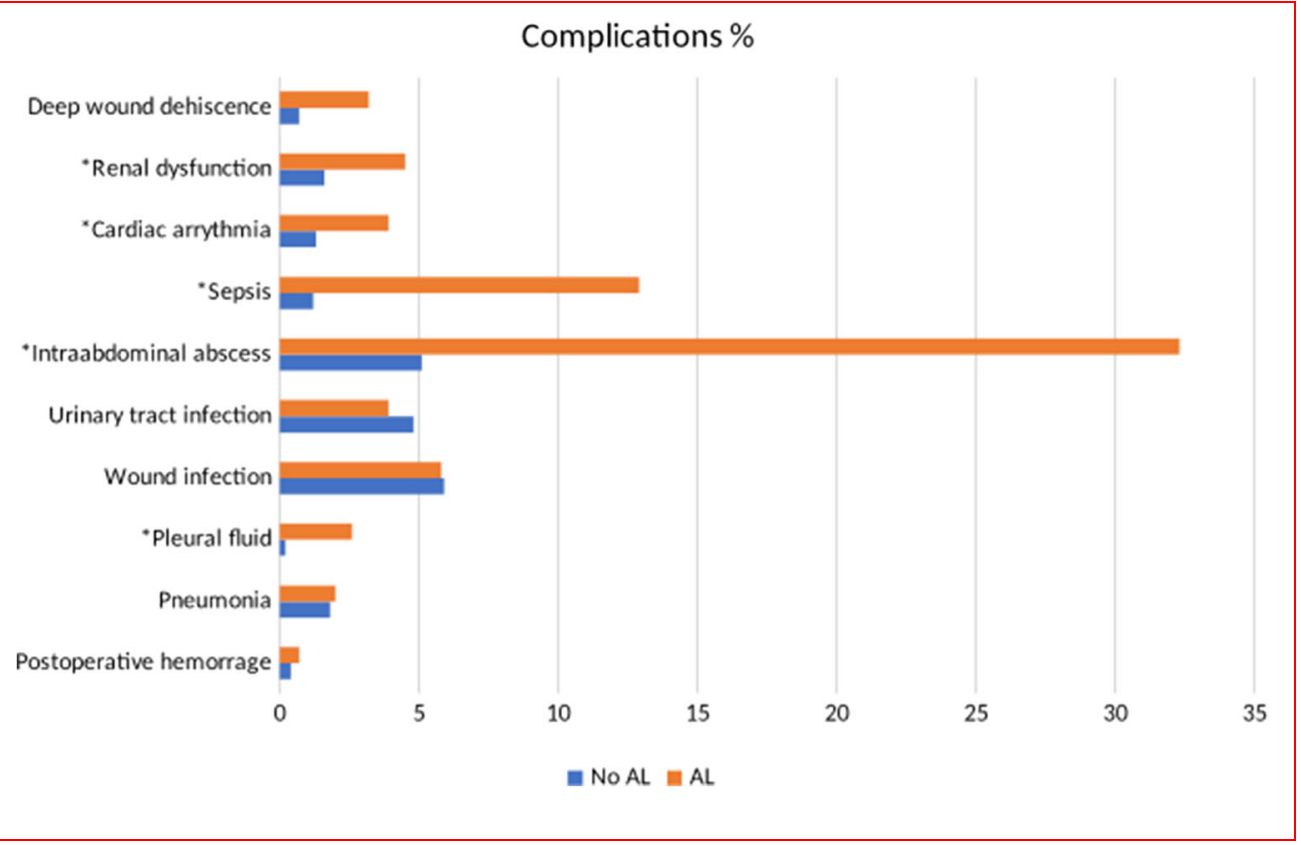

another recognized risk factor for AL [2, 8]. Suggested causal factors such as obesity, adverse intraoperative events, increased bacterial exposure and hypoperfusion might explain this association.

The finding that surgery late in the study period was associated with an increased rate of AL is unexpected since studies have shown that leak rates seem to be stable over time [21, 29]. We did not find a clear explanation for this finding, but possible explanations are that diagnostics with $\mathrm{X}$-ray investigations have increased over time, a more thorough registration of AL and increased use of neoadjuvant radiotherapy later in the study period. Older age was identified as a protective factor against AL in our study, not in line with data from studies showing results in the opposite direction [30, 31]. This discrepancy may be by chance since the effect, although statistically significant, was marginal. Preadmission education has never before been investigated in AL research. Previous studies show a possible impact on variables such as pain and anxiety $[32,33]$, but the association with $\mathrm{AL}$ in this study is more difficult to explain. Education might help the patients improve their conditions before surgery.

Other previously presented risk factors for AL such as smoking/alcohol consumption [34, 35], diabetes [36], pulmonary disease [37], high ASA fitness grade [38], poor preoperative nutritional status measured as weight loss or hypoalbuminemia [39, 40], preoperative chemotherapy or preoperative radiotherapy $[9,25,41]$ could not be identified as risk factors for $\mathrm{AL}$ in the current multivariate analysis.

Neither surgical approach (open, laparoscopic, robotic) nor conversion to open surgery were found to be risk factors for AL, the latter somewhat surprising since conversion have been described as a proxy for difficult surgery [42] and reported to result in higher complication rates [43], but not AL per se.

$\mathrm{AL}$ was, as expected, associated with increased shortterm morbidity, higher rate of reoperations and longer LOS. Our study did not demonstrate a difference in mortality between groups, but mortality may rise over 90 days and this may better reflect the true mortality related to the operation [7].

Even though increased compliance to the ERAS protocol has been shown to improve short-term outcome in previous studies [20], comparing pre- and intraoperative compliance in patients with and without $\mathrm{AL}$ in the current study revealed one care item-excess of IV volume of fluids intraoperatively, as a univariate predictor for AL. Perioperative overhydration has been associated with poor outcome and even increased risk for AL in studies [44, 45], although underhydration might also confer increased morbidity [46]. The finding that no other compliance item made a significant difference in univariate analysis may well be explained by the overall high compliance in both groups. Postoperative compliance measures, i.e., variables related to outcome from surgery, showed-as expectedworse results in the AL group.

The strength of the current study is the multicenter design, collecting over 300 prospectively and consecutively recorded variables from the ERAS database which makes it possible to study multiple important perioperative covariates that may to a larger extent reflect clinical reality compared to data collected in randomized trials. Also, by 
measuring compliance, we can control for the treatment before and during surgery.

On the other hand, the retrospective design makes it difficult to draw firm conclusions on outcome, and although we tried to minimize errors using multivariate analysis, unknown factors affecting the results cannot be ruled out. Such a limitation is time. During the 10-year long inclusion period, changes can occur in attitudes and turnover among staff in the ERAS program as well as technical progress. Despite that time was adjusted for in the multivariate analysis and compliance to the protocol was similar between groups over time, this time factor might be difficult to fully eliminate. A weakness in trying to study the role of compliance to ERAS as risk factor for AL is the uniform and high compliance to the protocol which reduce chances of detecting such a role. Another weakness is the lack of data on tumor height and level of anastomosis in EIAS, factors that are important for the risk of developing AL. Other drawbacks acknowledged are limitations in the register itself that could introduce bias, such as short follow-up time (30 days) and lack of data on postoperative pathology, e.g. staging.

In conclusion, in this large multicenter cohort study in hospitals with high compliance to an ERAS program, we found seven independent risk factors for AL: male gender, high BMI, year of surgery, peritoneal contamination, duration of surgery, age and preadmission education in patients operated on with anterior resection. The only protective compliance item remaining after adjustment for covariates was preadmission education. The absence of impact from overall compliance may in part be explained by high and uniform ERAS compliance in the participating centers of the study. Further studies should aim at preparing patients within an optimization program several weeks before major surgery, taking risk factors for AL into account.

Acknowledgements Collaborators: Representatives for all Swedish ERAS centers contributing with patients registered in the international ERAS database: Johan Carlander, MD, Department of Surgery, Västmanlands hospital, Västerås, Sweden. Dan Kornfeld, MD, Capio St Görans hospital, Stockholm, Sweden. Frida Olsson, Nurse Practitioner, Department of Surgical Sciences, Uppsala University, Akademiska Sjukhuset, Uppsala, Sweden. Malte Norström, MD, Department of Surgery, Kungälv hospital, Sweden. Lars Johansen MD, Department of Surgery, Skaraborg hospital, Skövde, Sweden. Sanna Laine, Nurse specialist, Department of Surgery, Karlstad hospital, Karlstad, Sweden. Markus Lindqvist, Nurse specialist, Department of Surgery, Norrland University hospital, Umeå, Sweden. Elin Holmstrand, Nurse specialist, Department of Surgery, Skaraborg hospital, Lidköping, Sweden. Tomas Koczkas, MD, Department of surgery, Östersund hospital. Johan Tranberg, MD, Östra hospital/ Sahlgrenska, Göteborg, Sweden. Monika Egenvall, MD, Karolinska Institute, Department of Molecular Medicine and Surgery and Division of Coloproctology, Center for Digestive Diseases, Karolinska University Hospital, Sweden.
Funding Open access funding provided by Karolinska Institute.

\section{Declarations}

Conflict of interest The first author, Daniel Asklid, the third author Yin $\mathrm{Xu}$ and the last author Ulf O Gustafsson declare no conflict of interest. The second author Olle Ljungqvist is the chairman and cofounder of the ERAS Society, and he is the founder and shareholder of Encare AB, a software company that develops the ERAS Interactive Audit System.

Ethics approval The study was approved by the Regional Ethical Review Board in Stockholm (2020-00435) and completed in accordance with the Declaration of Helsinki of the World Medical Association (1989).

Open Access This article is licensed under a Creative Commons Attribution 4.0 International License, which permits use, sharing, adaptation, distribution and reproduction in any medium or format, as long as you give appropriate credit to the original author(s) and the source, provide a link to the Creative Commons licence, and indicate if changes were made. The images or other third party material in this article are included in the article's Creative Commons licence, unless indicated otherwise in a credit line to the material. If material is not included in the article's Creative Commons licence and your intended use is not permitted by statutory regulation or exceeds the permitted use, you will need to obtain permission directly from the copyright holder. To view a copy of this licence, visit http://creativecommons. org/licenses/by/4.0/.

\section{References}

1. Gessler B, Eriksson O, Angenete E (2017) Diagnosis, treatment, and consequences of anastomotic leakage in colorectal surgery. Int J Colorectal Dis 32(4):549-556

2. Kawada K, Sakai Y (2016) Preoperative, intraoperative and postoperative risk factors for anastomotic leakage after laparoscopic low anterior resection with double stapling technique anastomosis. World J Gastroenterol 22(25):5718-5727

3. McDermott FD et al (2015) Systematic review of preoperative, intraoperative and postoperative risk factors for colorectal anastomotic leaks. Br J Surg 102(5):462-479

4. Pommergaard $\mathrm{HC}$ et al (2014) Preoperative risk factors for anastomotic leakage after resection for colorectal cancer: a systematic review and meta-analysis. Colorectal Dis 16(9):662-671

5. Mirnezami A et al (2011) Increased local recurrence and reduced survival from colorectal cancer following anastomotic leak: systematic review and meta-analysis. Ann Surg 253(5):890-899

6. Lu ZR et al (2016) Anastomotic leaks after restorative resections for rectal cancer compromise cancer outcomes and survival. Dis Colon Rectum 59(3):236-244

7. Bostrom P et al (2019) Population-based cohort study of the impact on postoperative mortality of anastomotic leakage after anterior resection for rectal cancer. BJS Open 3(1):106-111

8. Sciuto A et al (2018) Predictive factors for anastomotic leakage after laparoscopic colorectal surgery. World J Gastroenterol 24(21):2247-2260

9. $\mathrm{Hu} \mathrm{MH}$ et al (2017) Does neoadjuvant therapy increase the incidence of anastomotic leakage after anterior resection for mid and low rectal cancer? a systematic review and meta-analysis. Colorectal Dis 19(1):16-26

10. Zhang W et al (2017) Multicenter analysis of risk factors for anastomotic leakage after middle and low rectal cancer resection 
without diverting stoma: a retrospective study of 319 consecutive patients. Int J Colorectal Dis 32(10):1431-1437

11. Wu SW, Ma CC, Yang Y (2014) Role of protective stoma in low anterior resection for rectal cancer: a meta-analysis. World $\mathrm{J}$ Gastroenterol 20(47):18031-18037

12. Ljungqvist O, Scott M, Fearon KC (2017) Enhanced recovery after surgery: a review. JAMA Surg 152(3):292-298

13. Gustafsson UO et al (2019) Guidelines for perioperative care in elective colorectal surgery: enhanced recovery after surgery (ERAS(R)) society recommendations: 2018. World J Surg 43(3):659-695

14. Ban KA, Berian JR, Ko CY (2019) Does implementation of enhanced recovery after surgery (ERAS) protocols in colorectal surgery improve patient outcomes? Clin Colon Rectal Surg 32(2):109-113

15. Maessen $\mathbf{J}$ et al (2007) A protocol is not enough to implement an enhanced recovery programme for colorectal resection. Br J Surg 94(2):224-231

16. Dindo D, Hahnloser D, Clavien PA (2010) Quality assessment in surgery: riding a lame horse. Ann Surg 251(4):766-771

17. Bursac $\mathrm{Z}$ et al (2008) Purposeful selection of variables in logistic regression. Source Code Biol Med 3:17

18. Demidenko E (2007) Sample size determination for logistic regression revisited. Stat Med 26(18):3385-3397

19. White IR, Royston P, Wood AM (2011) Multiple imputation using chained equations: issues and guidance for practice. Stat Med 30(4):377-399

20. Gustafsson UO et al (2011) Adherence to the enhanced recovery after surgery protocol and outcomes after colorectal cancer surgery. Arch Surg 146(5):571-577

21. van Rooijen SJ et al (2016) Intraoperative modifiable risk factors of colorectal anastomotic leakage: why surgeons and anesthesiologists should act together. Int J Surg 36(Pt A):183-200

22. Rahbari NN et al (2010) Definition and grading of anastomotic leakage following anterior resection of the rectum: a proposal by the international study group of rectal cancer. Surgery 147(3):339-351

23. Kulu Y et al (2013) Validation of the international study group of rectal cancer definition and severity grading of anastomotic leakage. Surgery 153(6):753-761

24. Borstlap WAA et al (2017) Anastomotic leakage and chronic presacral sinus formation after low anterior resection: results from a large cross-sectional study. Ann Surg 266(5):870-877

25. Park JS et al (2013) Multicenter analysis of risk factors for anastomotic leakage after laparoscopic rectal cancer excision: the Korean laparoscopic colorectal surgery study group. Ann Surg 257(4):665-671

26. Sparreboom CL et al (2018) Different risk factors for early and late colorectal anastomotic leakage in a nationwide audit. Dis Colon Rectum 61(11):1258-1266

27. Ba ZF et al (2004) Gender differences in small intestinal endothelial function: inhibitory role of androgens. Am J Physiol Gastrointest Liver Physiol 286(3):G452-G457

28. Konishi $\mathrm{T}$ et al (2006) Risk factors for anastomotic leakage after surgery for colorectal cancer: results of prospective surveillance. J Am Coll Surg 202(3):439-444

29. Meyer $\mathbf{J}$ et al (2019) Reducing anastomotic leak in colorectal surgery: the old dogmas and the new challenges. World J Gastroenterol 25(34):5017-5025

30. Chan DKH et al (2020) Age is an independent risk factor for increased morbidity in elective colorectal cancer surgery despite an ERAS protocol. Langenbecks Arch Surg 405(5):673-689
31. Aquina CT et al (2017) The impact of age on complications, survival, and cause of death following colon cancer surgery. Br J Cancer 116(3):389-397

32. Guo P, East L, Arthur A (2012) A preoperative education intervention to reduce anxiety and improve recovery among Chinese cardiac patients: a randomized controlled trial. Int J Nurs Stud 49(2):129-137

33. Sjoling M et al (2003) The impact of preoperative information on state anxiety, postoperative pain and satisfaction with pain management. Patient Educ Couns 51(2):169-176

34. Richards $\mathrm{CH}$ et al (2012) Smoking is a major risk factor for anastomotic leak in patients undergoing low anterior resection. Colorectal Dis 14(5):628-633

35. Sorensen LT et al (1999) Smoking and alcohol abuse are major risk factors for anastomotic leakage in colorectal surgery. Br J Surg 86(7):927-931

36. Cong ZJ et al (2009) Influencing factors of symptomatic anastomotic leakage after anterior resection of the rectum for cancer. World J Surg 33(6):1292-1297

37. Rencuzogullari A et al (2017) Predictors of anastomotic leak in elderly patients after colectomy: nomogram-based assessment from the American college of surgeons national surgical quality program procedure-targeted cohort. Dis Colon Rectum 60(5):527-536

38. Pucciarelli S et al (2019) Multicentre randomized clinical trial of colonic $\mathrm{j}$ pouch or straight stapled colorectal reconstruction after low anterior resection for rectal cancer. $\mathrm{Br} \quad \mathrm{J}$ Surg 106(9):1147-1155

39. Yamano $\mathrm{T}$ et al (2016) Malnutrition in rectal cancer patients receiving preoperative chemoradiotherapy is common and associated with treatment tolerability and anastomotic leakage. Int $\mathbf{J}$ Colorectal Dis 31(4):877-884

40. Suding P et al (2008) Definitive risk factors for anastomotic leaks in elective open colorectal resection. Arch Surg. https://doi.org/ 10.1001/archsurg.143.9.907

41. Qu H, Liu Y, Bi DS (2015) Clinical risk factors for anastomotic leakage after laparoscopic anterior resection for rectal cancer: a systematic review and meta-analysis. Surg Endosc 29(12):3608-3617

42. Baek JH et al (2010) Oncologic outcomes of robotic-assisted total mesorectal excision for the treatment of rectal cancer. Ann Surg 251(5):882-886

43. Guillou PJ et al (2005) Short-term endpoints of conventional versus laparoscopic-assisted surgery in patients with colorectal cancer (MRC CLASICC trial): multicentre, randomised controlled trial. Lancet 365(9472):1718-1726

44. Boesen AK, Maeda Y, Rorbaek Madsen M (2013) Perioperative fluid infusion and its influence on anastomotic leakage after rectal cancer surgery: implications for prevention strategies. Colorectal Dis. https://doi.org/10.1111/codi.12321

45. Asklid D et al (2017) The impact of perioperative fluid therapy on short-term outcomes and 5-year survival among patients undergoing colorectal cancer surgery-a prospective cohort study within an ERAS protocol. Eur J Surg Oncol 43(8):1433-1439

46. Myles PS et al (2018) Restrictive versus liberal fluid therapy for major abdominal surgery. N Engl J Med 378(24):2263-2274

Publisher's Note Springer Nature remains neutral with regard to jurisdictional claims in published maps and institutional affiliations. 